\title{
Three dimensional intruder closest point of approach estimation based-on monocular image parameters in aircraft sense and avoid
}

\author{
Motto: 'Almost everything from almost nothing'
}

\author{
Peter Bauer • Antal Hiba • Jozsef Bokor . \\ Akos Zarandy
}

Received: date / Accepted: date

\begin{abstract}
The paper deals with monocular image-based sense and avoid assuming constant aircraft velocities and straight flight paths. From very limited two dimensional image information it finally characterizes the whole three dimensional collision situation by estimating the time to closest point of approach, the horizontal relative distance and its direction and the vertical relative distance also. The distances are relative to the intruder aircraft horizontal and vertical sizes. The overall estimated relative distance is the closest between the two aircraft in three dimension. So finally, every important information can be extracted to be used in a collision decision. The applicability of the developed method is presented in software-in-the-loop simulation test runs. Several intruder size and speed values are considered together with trajectories covering the whole three dimensional space. The horizontal intruder flight directions relative to the own aircraft cover
\end{abstract}

The research leading to these results has received funding from the European Union's Horizon 2020 research and innovation programme under grant agreement No. 690811 and the Japan New Energy and Industrial Technology Development Organization under grant agreement No. 062600 as a part of the EU/Japan joint research project entitled 'Validation of Integrated Safety-enchanced Intelligent flight cONtrol (VISION)' This work was also supported by the Institute for Computer Science and Control (SZTAKI) Grant Number 008

P. Bauer

Systems and Control Laboratory, MTA SZTAKI, H-1111 Budapest, Kende utca 13-17.

(ORCID ID: 0000-0002-1925-2270)

Tel.: +36-1-2796163

Fax: +36-1-4667483

E-mail: bauer.peter@sztaki.mta.hu

A. Hiba

Computational Optical Sensing and Processing Laboratory, MTA SZTAKI, H-1111 Budapest, Kende utca 13-17.

(ORCID ID: 0000-0002-5706-3550)

J. Bokor

Systems and Control Laboratory, MTA SZTAKI, H-1111 Budapest, Kende utca 13-17. (ORCID ID: 0000-0002-3461-4807)

\section{A. Zarandy}

Computational Optical Sensing and Processing Laboratory, MTA SZTAKI, H-1111 Budapest, Kende utca 13-17. 
$360^{\circ}$ and the intruder can come from below ar above also. Detailed evaluation and discussion of the results is also included. Finally, the missed detection rate results to be superior (below $3 \%$ in every test scenario) though the false alarm rate results a bit high between $7-14 \%$.

Keywords Sense and avoid · Monocular camera - Closest point of approach . Intruder direction

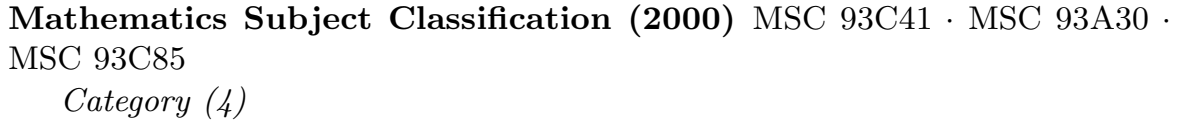

\section{Introduction}

Sense and avoid (S\&A) capability is a crucial ability for the future unmanned aerial vehicles (UAVs). It is vital to integrate civilian and governmental UAVs into the common airspace according to [9] for example. At the highest level of integration Airborne Sense and Avoid (ABSAA) systems are required to guarantee airspace safety [8].

In this field the most critical question is the case of non-cooperative S\&A for which usually complicated multi-sensor systems (see [12], [23] for example) or radar-based solutions (see [11] and [15] for example) are developed. However, in case of small UAVs the size, weight and power consumption of the onboard S\&A system should be minimal. Monocular vision-based solutions can be cost and weight effective therefore especially good for small UAVs [7], [13], [16], [17], [20], [21] [22], [27]. These systems basically measure the position (bearing) and size of intruder aircraft $(\mathrm{A} / \mathrm{C})$ camera image without range and intruder size information. This scale ambiguity makes the decision about the possibility of collision complicated. The first approach of the authors and colleagues to overcome this difficulty was to apply artificial stereo vision by maneuvering the own aircraft [14], [26], [28], [30]. This approach has two drawbacks, the first is the loss of energy efficiency because of the required extra maneuvering, the second is the limitation of the effective field of view of the camera system (see [5]). However, [7] points out that the relative distance of intruder from own $\mathrm{A} / \mathrm{C}$ (when it crosses the camera focal plane) called closest point of approach (CPA) well characterizes the possibility of collision together with the time to closest point of approach (TTCPA) and there is a chance to estimate these parameters without additional maneuvers. In the literature there are several works dealing with TTCPA estimation based-on optic flow or first order motion models such as [6], [19], [24] however, it is hard to find references for CPA estimation ([24] makes a decision about the collision based-on the epipole position). Consequently, previous efforts of the authors [2], [3], [4] focused on the estimation of TTCPA and CPA values together with the direction of CPA from solely the monocular image parameters in the horizontal plane. In these previous works we referenced TTCPA as TTC (time to collision) however TTCPA better describes the meaning of this parameter. Formulae to obtain these values with forward looking and even with oblique cameras were developed and tested in software-in-the-loop (SIL) simulations and even in real flight. The proposed solution is a least squares (LS) optimal line fit based very simple algorithm which well 
fits real-time execution needs. Error analysis considering image pixelization errors was done in [4].

After successful implementation and flight testing onboard small UAVs (see [29] for preliminary results and our Youtube / AeroGNC channel for test flight and simulation videos) the authors attention turned towards omnidirectional camera systems with possibly $360^{\circ}$ horizontal field of view (FOV) (see [2], [3]). Such a system can be crucial in case of integration of small UAVs into the common airspace. Though Federal Aviation Administration (FAA) rules (FAR Part 91.113) regarding general aviation (GA) define that in case of overtaking 'Each aircraft that is being overtaken has the right-of-way and each pilot of an overtaking aircraft shall alter course to the right to pass well clear' (see e. g. [10]). It is well known that it is really hard for a GA pilot to observe a small size UAV so in this case his/her responsibility should be withdrawn and the UAV should provide clearance. [25] explicitly requires $360^{\circ}$ horizontal field of regard from a sense and avoid camera system so a multi-camera omnidirectional system covering $360^{\circ}$ horizontal field of view (FOV) is required. The theoretical basis of this topic is given by the handling of oblique camera setups [2]. Preliminary tests showed that the selection of the orientation of the reference body coordinate system influences TTCPA and CPA estimation. The optimal solution of this problem in the horizontal plane was the main topic of [3] together with extensive SIL testing and test on real flight data also.

However, the evaluation of the collision situation should be done in the vertical plane also as two aircraft can be very close in the horizontal plane though very far vertically which means a clear situation (no chance of collision). The extension of the formulae derived in [2], [3] and [4] into the vertical dimension is the first topic of this article. If one can evaluate the situation both in the horizontal and vertical planes the question arise if the three dimensional (3D) minimum distance between the aircraft can be determined from these parameters. This is the second topic of this article.

Finally, the whole 3D collision situation can be characterized in case of straight flight paths and constant flight velocities considering solely the monocular intruder image size and position information. The 3D TTCPA and horizontal $\left(C P A_{x}\right)$ and vertical $\left(C P A_{y}\right) \mathrm{CPA}$ values and their direction can be determined based-on the derived formulae. So one will know when and where will be the intruder closest to us. The only information lost is the absolute distance between the aircraft, the relative CPA values can be determined instead but this information is appropriate to decide about the possibility of collision. The required amount of information can be extracted from the monocular images which are well known to include scale ambiguity and so basically seems to make it impossible to decide about the collision situation. This means one can get almost everything from almost nothing. The structure of the article is as follows.

Section 2 summarizes the previous developments of the authors in the horizontal plane and so lays down the basics of further developments and integrates the notations. Section 3 extends the formulae into the vertical plane discussing also the related problems with the projection of an aircraft-like shape in the vertical plane. Section 4 presents some trajectory-based calculations pointing out that the horizontal and vertical smallest distances realize in different time instants and so do not give also the $3 \mathrm{D}$ minimum. The section also shows that the $3 \mathrm{D}$ minimum can be obtained by simple linear interpolation. The next section 5 proposes an 
interpolation between the horizontal and vertical CPA points (obtained in sections 2 and 3 ) to get the TTCPA and CPA values of the real 3D minimum. In section 6 the applied SIL test environment is briefly described and the results of a Monte-Carlo test campaign are presented. Finally, section 7 concludes the paper.

\section{Horizontal formulae for TTCPA and CPA estimation}

Consider first a close approach situation between two aicraft flying on straight paths with constant velocities in the horizontal plane (see Fig. 1).

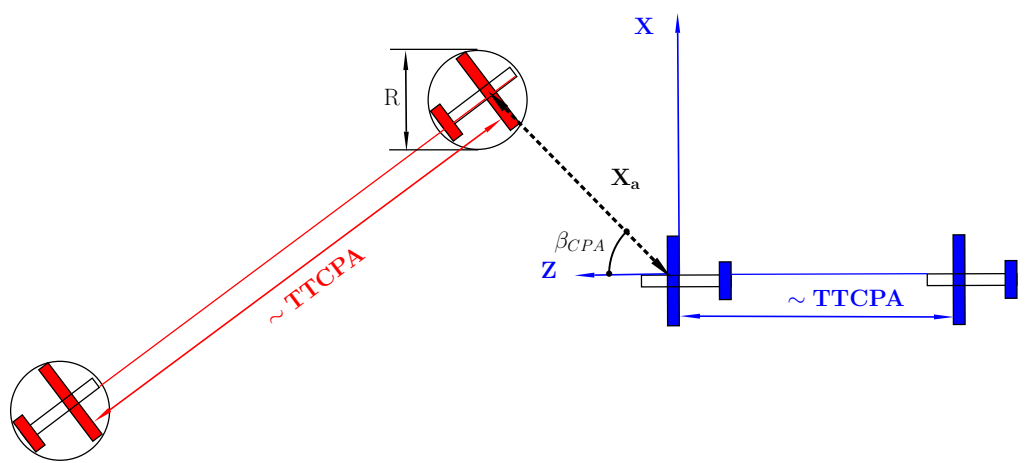

Fig. 1 Define TTCPA, $C P A=X_{a} / R$ and $\beta_{C P A}$ (intruder red from left, own aircraft blue from right)

The figure shows that TTCPA is the time when the two aicraft gets closest to each other. The closest distance between them is denoted by $X_{a}$ in the horizontal plane (will be denoted by $Y_{a}$ in the vertical plane). CPA is defined as the ratio of this distance and the characteristic horizontal size $(\mathrm{R})$ of the intruder. Usually the closest distance is not on the $\mathrm{X}$ axis of the own aircraft body coordinate system rather it is at a given direction characterized by $\beta_{C P A}$. So to evaluate the horizontal collision situation one has to determine $t_{C P A / x}, C P A_{x / x}$ and $\beta_{C P A / x}$. Here the subscript $x$ denotes that it is a horizontal parameter, while the second subscript $/ x$ denotes that the parameter is determined by looking for the horizontal minimum distance.

In the horizontal plane the aircraft can be modelled as a disc as the wingspan / fuselage length ratio of most of the aircraft is around 1 (see Fig. 17 in appendix A). Considering that the camera of the sense and avoid system can be oblique relative to the body coordinate system the disc projection model of the sense and avoid situation is shown in Fig. 2. In the figure (X,Z) is the (aircraft) body frame and $\left(X_{C}, Z_{C}\right)$ is the camera frame rotated by $\beta_{C}$ angle relative to the body. The disc represents the intruder aircraft (with half size $r=R / 2$ ) (original derivation of the disc projection model can be found in [4]). Before deriving the details of the projection model the possible measurable parameters of the intruder image in the image plane should be defined in Fig. 3 . 


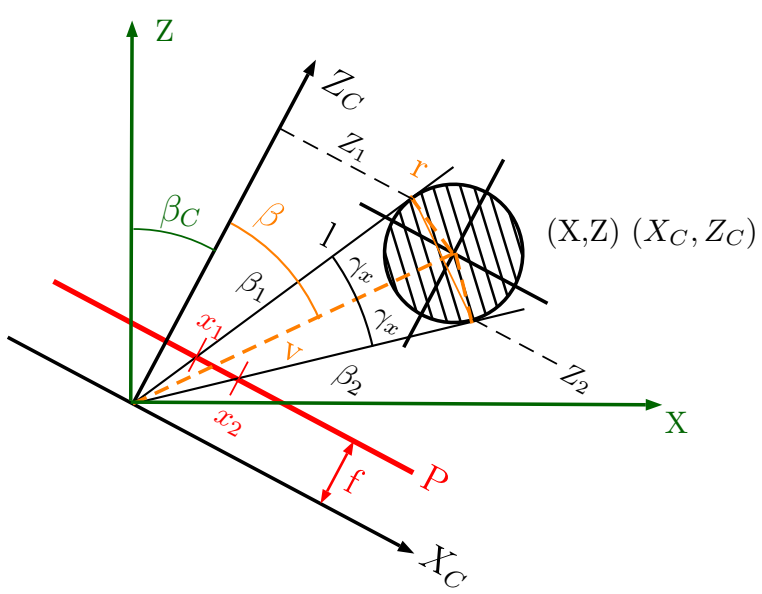

Fig. 2 Oblique camera disc projection model

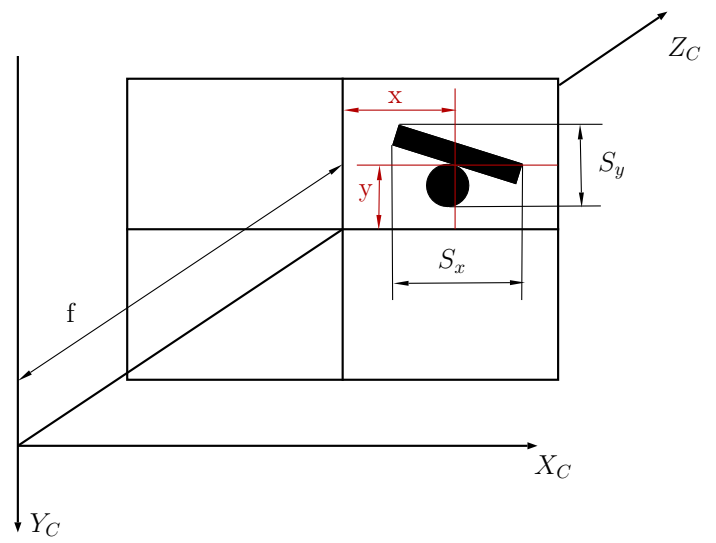

Fig. 3 Measurable image parameters

In $X_{C}, Y_{C}, Z_{C}$ camera frame $x, y$ are the positions of intruder image centroid, $S_{x}, S_{y}$ are the intruder image sizes (horizontal / vertical) and $f$ is the camera focal length. In the disc projection model (see Fig. 2) $S_{x}=x_{2}-x_{1}, x=\left(x_{2}+\right.$ $\left.x_{1}\right) / 2$ and $\beta_{1}=\operatorname{atan}\left(x_{1} / f\right), \quad \beta_{2}=\operatorname{atan}\left(x_{2} / f\right)$ can be defined as additional measurable image parameters. Unfortunately $x$ and $S_{x}$ can not be directly related to intruder distances $X_{C}, Z_{C}$ from the own aircraft in the camera frame. However, their transformed values $\bar{S}_{x}, \bar{x}$ has a close connection to $X_{C}, Z_{C}$ as shown in (1) (for details see [4]).

$$
\begin{aligned}
& \bar{S}_{x}=S_{x}\left(\cos \beta_{1}+\cos \beta_{2}\right)=\frac{2 f R}{Z_{C}} \\
& \bar{x}=x\left(1-\frac{\bar{S}_{x}^{2}}{16 f^{2}}\right)=f \frac{X_{C}}{Z_{C}}
\end{aligned}
$$


Considering now the $(\mathrm{X}, \mathrm{Y}, \mathrm{Z})$ intruder coordinates in the body frame characterized by the $X_{a}, Y_{a}$ miss distances (see Fig. 1 for example), the $V_{x}, V_{y}, V_{z}$ relative velocities and $t_{C P A}$ (equivalent to TTCPA) and executing the body to camera frame transformation one gets the $X_{C}, Y_{C}, Z_{C}$ coordinates. $Y_{a}$ is defined similarly as $X_{a}$ but in the $Y, Z$ plane. The $Y$ coordinate equations will be considered in the next section in the vertical plane formulae.

$$
\begin{aligned}
& X=X_{a}-V_{x} t_{C P A}, \quad Y=Y_{a}-V_{y} t_{C P A}, \quad Z=-V_{z} t_{C P A} \\
& X_{C}=X_{a} \cos \beta_{C}-\left(V_{x} \cos \beta_{C}-V_{z} \sin \beta_{C}\right) t_{C P A} \\
& Y_{C}=Y_{a}-V_{y} t_{C P A} \\
& Z_{C}=X_{a} \sin \beta_{C}-\left(V_{x} \sin \beta_{C}+V_{z} \cos \beta_{C}\right) t_{C P A}
\end{aligned}
$$

Substituting now the expressions of $X_{C}$ and $Z_{C}$ into the reciprocal and ratio of the expressions for $\bar{x}$ and $\bar{S}_{x}$ in (1) and considering $C P A_{x}=\frac{X_{a}}{R}$ one gets:

$$
\begin{aligned}
& \frac{1}{\bar{S}_{x}}=\frac{C P A_{x}}{2} \frac{\sin \beta_{C}}{f}-\frac{V_{x} \sin \beta_{C}+V_{z} \cos \beta_{C}}{2 f R} t_{C P A} \\
& \frac{\bar{x}}{\bar{S}_{x}}=\frac{C P A_{x}}{2} \cos \beta_{C}-\frac{V_{x} \cos \beta_{C}-V_{z} \sin \beta_{C}}{2 R} t_{C P A}
\end{aligned}
$$

In this system of equations the unknowns are $C P A_{x}$ and $t_{C P A}$ and the time varying terms are $\bar{x}, \bar{S}_{x}, t_{C P A}$. The other terms such as the camera focal length $f$, the camera angle $\beta_{C}$, the relative velocities $V_{x}, V_{z}$ and the intruder size $R$ are all constant. Considering this and $t_{C P A}=t_{C}-t$ one gets $\left(t\right.$ is actual time, $t_{C}$ is the time when intruder is closest to own aircraft (it is constant)):

$$
\begin{aligned}
& \frac{1}{\bar{S}_{x}}=\frac{\sin \beta_{C}}{f} \frac{C P A_{x}}{2}-a_{1} t_{C}+a_{1} t=c_{1}+a_{1} t \\
& \frac{\bar{x}}{\bar{S}_{x}}=\cos \beta_{C} \frac{C P A_{x}}{2}-a_{2} t_{C}+a_{2} t=c_{2}+a_{2} t
\end{aligned}
$$

Fitting least squares (LS) optimal linear curves to the expressions on the left side of (4) with independent variable $t$ gives $a_{1}, a_{2}, c_{1}, c_{2}$. This fits require at least two data points but possibly 8-10 points should be used to suppress the effect of pixelization and other image errors. From the estimated coefficients a system of linear equations results for $C P A_{x} / 2$ and $t_{C}$ :

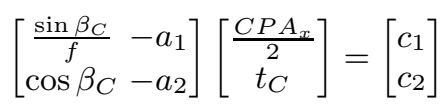

Solvability of this system of equations is proven in [2] however, the $C P A_{x}$ solution will give the closest point of approach when the intruder crosses the $\mathrm{X}$ axis of the body coordinate frame in Fig. 2 and this is not always the smallest $C P A_{x}$ in the horizontal plane as [3] points out in details. Usually the body system should be rotated by a $-\Delta \beta_{C / x}$ angle to have an orientation which gives $\beta_{C P A}$ and the real minimum $C P A_{x}$. This gives a different $\beta_{C / x}^{\prime}=\beta_{C}+\Delta \beta_{C / x}$ camera angle (the camera is assumed to be fixed). Reformulating now (5) with the new angle gives: 


$$
\begin{aligned}
& {\left[\begin{array}{cc}
\frac{\sin \beta_{C / x}^{\prime}}{f}-a_{1} \\
\cos \beta_{C / x}^{\prime}-a_{2}
\end{array}\right]\left[\begin{array}{c}
\frac{C P A_{x}}{2} \\
t_{C}
\end{array}\right]=\left[\begin{array}{l}
c_{1} \\
c_{2}
\end{array}\right]} \\
& {\left[\begin{array}{cc}
\frac{\sin \left(\beta_{C}+\Delta \beta_{C / x}\right)}{f} & -a_{1} \\
\cos \left(\beta_{C}+\Delta \beta_{C / x}\right) & -a_{2}
\end{array}\right]\left[\begin{array}{c}
\frac{C P A_{x}}{2} \\
t_{C}
\end{array}\right]=\left[\begin{array}{l}
c_{1} \\
c_{2}
\end{array}\right]}
\end{aligned}
$$

According to (4) parameters $a_{1}, a_{2}, c_{1}, c_{2}$ are constant in a given camera configuration irrespective of the orientation of the body frame. So, minimization of $C P A_{x}$ by the proper choice of $\Delta \beta_{C / x}$ is possibly feasible. A detailed derivation in [3] gives a closed form easily calculable solution:

$$
\tan \Delta \beta_{C / x}=\frac{\left(\frac{-a_{2} \cos \beta_{C}}{f}-a_{1} \sin \beta_{C}\right)}{\left(\frac{-a_{2} \sin \beta_{C}}{f}+a_{1} \cos \beta_{C}\right)}
$$

By substituting $\Delta \beta_{C / x}$ back to (6) one gets the real minimum $C P A_{x / x}$ solution and the related $t_{C / x}$ and $t_{C P A / x}$ value and finally $\beta_{C P A / x}$ also (for details see $[3])$ :

$$
\beta_{C P A / x}=-\Delta \beta_{C / x}+\operatorname{sign}\left(C P A_{x / x}\right) \frac{\pi}{2}
$$

In summary, it can be stated that TTCPA, global minimum CPA and direction of CPA $\left(\beta_{C P A}\right)$ can all be calculated in the horizontal plane based solely on the image parameters obtained from a monocular camera system.

\section{Vertical formulae for TTCPA and CPA estimation}

After deriving formulae to determine the CPA in the horizontal plane the next step can be to do the same in the vertical plane. In the vertical plane the aircraft can not be modelled as a disc its rather similar to an ellipse (length almost equal to the horizontal size of the intruder, height equal to the height of the intruder). It can be shown that the pinhole camera projection model of the ellipse is very complicated thus an extension to the same disc as in the horizontal plane can be done if the horizontal size is transformable to the vertical plane. The situation is shown in Fig. 4. As there is no point in using vertically oblique cameras in this model the camera points horizontally forward. However, as the camera can be oblique in the horizontal plane this means that the body $Z$ axis and the camera $Z_{C}$ axis will not coincide trivially. That's why only the camera $Z_{C}$ axis is shown in the figure. On the other hand the body $Y$ and camera $Y_{C}$ axes can be assumed to coincide as any rotation of the body system in the vertical plane should not be considered (see later). 


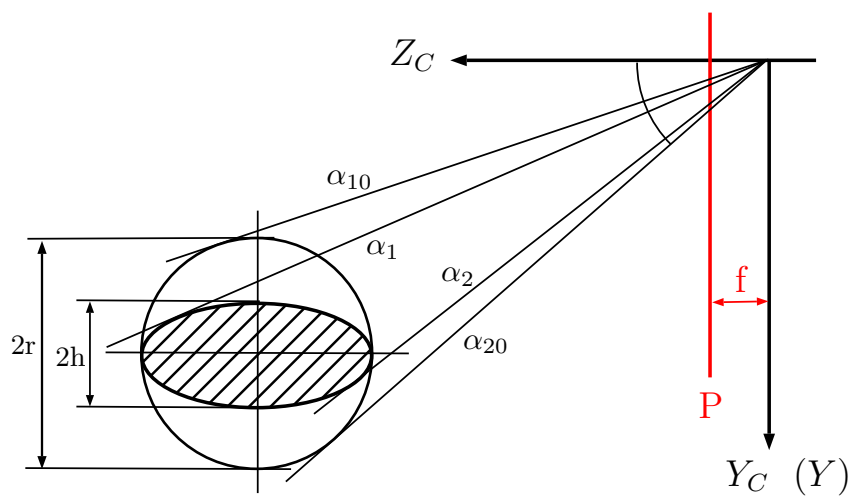

Fig. 4 Vertical camera ellipse and disc projection models

The extension to disc projection can be trivially done by symmetrically placing the view angle range of the disc around the view angle range of the ellipse. This requires the differences $\left|\alpha_{10}-\alpha_{1}\right|$ and $\left|\alpha_{20}-\alpha_{2}\right|$ to be almost equal considering the projections in Fig. 4.

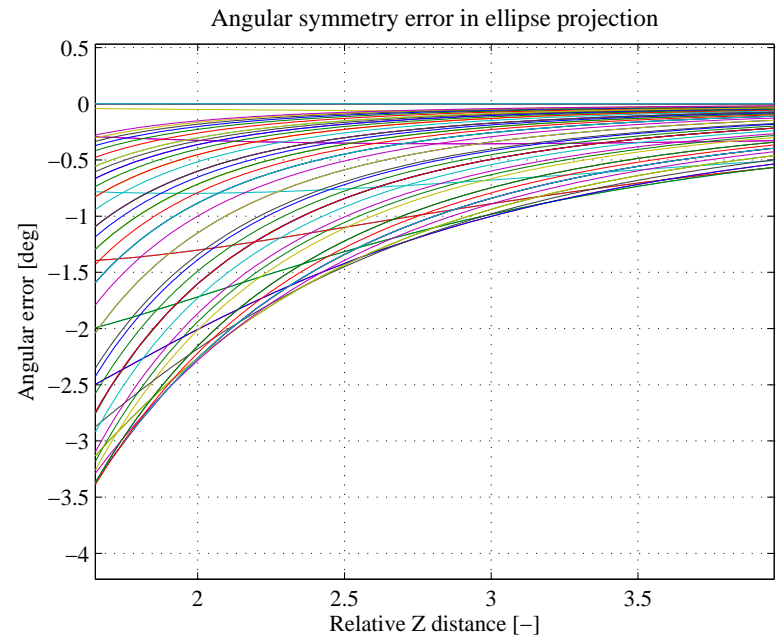

Fig. 5 Angular symmetry error between ellipse and disc

The difference between these absolute differences is plotted in Fig. 5 for $Z=$ $1000: 10 m Y=[0,5,10,20,50,100] m$ and vertical $H=2 h=1 m$ and horizontal $R=2 r=5 m$ basic sizes scaled from 1 to 12 . This $1 / 5=0.2$ ratio is about the minimum height/average size ratio of the aircraft (see Fig. 18) and so results in the most difference between the angles. A higher ratio approximates the disc better and so should give smaller angular differences. That's why the curves are plotted for the minimum ratio. This means that the differences are plotted for $H=1-12 \mathrm{~m}$ and $R=5-60 \mathrm{~m}$. All the angular differences are plotted against 
$\frac{Z}{R}$ to make the curves for different $R$ sizes comparable. The results show that for $\frac{Z}{R} \geq 3$ the angular difference is below $1^{\circ}$ and so the symmetric approximation will be surely valid until this point which means an intruder in a distance from the own aircraft at 3 times its characteristic size. Below this distance the NMAC is obvious and so there is no point in using the S\&A system (its too late). These calculations show that virtual projection of the horizontal size as a disc in the vertical plane is possible by symmetrically extending the measured vertical size. This will give the same image position but a similar size to the horizontal one.

Simple use of the same image size as $S_{x}$ in the horizontal plane can be misleading as the projected size of the disc changes depending on its view angle (see Fig. 6). So a size correction should be done before projecting to the vertical plane considering the horizontal $\beta$ (see Fig. 2) and vertical $\alpha$ (see Fig. 4) view angles.

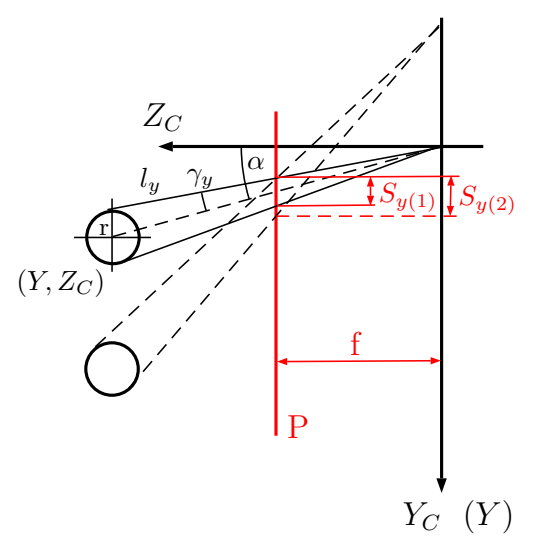

Fig. 6 Change of disc projected size with view angle

$$
\begin{aligned}
& l_{y}=\sqrt{Y^{2}+Z_{C}^{2}-r^{2}} \\
& \tan \left(\gamma_{y}\right)=\frac{r}{l_{y}} \\
& \tan \alpha=\frac{Y}{Z_{C}} \rightarrow Y=Z_{C} \tan \alpha \\
& \tan \left(\gamma_{y}\right)=\frac{r}{\sqrt{Z_{C}^{2}\left(1+\tan ^{2} \alpha\right)-r^{2}}} \\
& \tan \left(\gamma_{x}\right)=\frac{r}{l_{x}}=\frac{r}{\sqrt{Z_{C}^{2}\left(1+\tan ^{2} \beta\right)-r^{2}}} \\
& \frac{\tan \left(\gamma_{y}\right)}{\tan \left(\gamma_{x}\right)}=\frac{\sqrt{Z_{C}^{2}\left(1+\tan ^{2} \beta\right)-r^{2}}}{\sqrt{Z_{C}^{2}\left(1+\tan ^{2} \alpha\right)-r^{2}}} \\
& \text { if } Z_{C} \gg r \\
& \tan \left(\gamma_{y}\right)=\frac{\sqrt{1+\tan ^{2} \beta}}{\sqrt{1+\tan ^{2} \alpha}} \tan \left(\gamma_{x}\right)
\end{aligned}
$$


Fig. 6 shows the vertical situation with view angle $\alpha$ and half subtended angle $\gamma_{y}$. In the horizontal plane Fig. 2 shows the same with view angle $\beta$ and half subtended angle $\gamma_{x}$. Considering the calculation of the half subtended angles an approximate transformation formula can be derived in (9).

$Z_{C} \gg r$ is satisfied by $\frac{Z}{R} \geq 3 \rightarrow \frac{Z}{r} \geq 6$ which is the limit of validity in the previous step. From equation (9) the steps of the virtual vertical disc size calculation are as follows:

1. Determine the half subtended angle $\gamma_{x}$ and the view angle $\beta$ in the horizontal plane

2. Determine the view angle $\alpha$ in the vertical plane

3. Determine the required vertical half subtended angle from:

$$
\tan \left(\gamma_{y}\right)=\frac{\sqrt{1+\tan ^{2} \beta}}{\sqrt{1+\tan ^{2} \alpha}} \tan \left(\gamma_{x}\right)
$$

4. Determine the virtual vertical size as: $S_{y}^{\prime}=f\left(\tan \left(\alpha+\gamma_{y}\right)-\tan \left(\alpha-\gamma_{y}\right)\right)$. This will be related to the real horizontal size $R=2 r$ of the intruder.

From this point the vertical projection formulae for the virtual disc result analogously to the $\mathrm{X}-\mathrm{Z}$ horizontal ones but by considering the $Y$ distances shown in (2) in the Y-Z plane and defining the vertical relative closest point of approach with respect to the virtual disc size:

$$
C P A_{y}^{\prime}=\frac{Y_{a}}{R}=\frac{Y_{a}}{2 r}
$$

The transformed virtual vertical size and position of the intruder image gives:

$$
\begin{aligned}
& \bar{S}_{y}^{\prime}=S_{y}^{\prime}\left(\cos \alpha_{1}+\cos \alpha_{2}\right)=\frac{2 f R}{Z_{C}} \\
& \bar{y}=y\left(1-\frac{\bar{S}_{y}^{\prime 2}}{16 f^{2}}\right)=f \frac{Y_{C}}{Z_{C}}
\end{aligned}
$$

The reciprocal value of $\bar{S}_{y}^{\prime}$ and its ratio with $\bar{y}$ together with the substitution of the distances from (2) gives:

$$
\begin{aligned}
\frac{1}{\bar{S}_{y}^{\prime}} & =\frac{C P A_{x}}{2} \frac{\sin \beta_{C}}{f}-\frac{\left(V_{x} \sin \beta_{C}+V_{z} \cos \beta_{C}\right) t_{C P A}}{2 f R} \\
\frac{\bar{y}}{\bar{S}_{y}^{\prime}} & =\frac{C P A_{y}^{\prime}}{2}-\frac{V_{y}}{2 R} t_{C P A}
\end{aligned}
$$

The first equation is exactly the same as in the horizontal plane in (3) so the mean value of the two equations can be considered in (4) to determine $C P A_{x / x}$ and $t_{C P A / x}$. This again means the determination of the horizontal plane parameters, the estimation of the vertical parameters is discussed later. 


$$
\begin{aligned}
& \frac{1}{2}\left(\frac{1}{\bar{S}_{x}}+\frac{1}{\bar{S}_{y}^{\prime}}\right)=\frac{C P A_{x} \sin \beta_{C}}{2 f}-\frac{\left(V_{x} \sin \beta_{C}+V_{z} \cos \beta_{C}\right)}{2 R f} t_{C P A} \\
& \frac{1}{2}\left(\frac{1}{\bar{S}_{x}}+\frac{1}{\bar{S}_{y}^{\prime}}\right)=\frac{\sin \beta_{C}}{f} \frac{C P A_{x}}{2}-a_{1} t_{C}+a_{1} t=c_{1}+a_{1} t \\
& \frac{\bar{x}}{\bar{S}_{x}}=\cos \beta_{C} \frac{C P A_{x}}{2}-a_{2} t_{C}+a_{2} t=c_{2}+a_{2} t
\end{aligned}
$$

The mean should be considered because of possible numerical errors in calculating $\bar{S}_{y}^{\prime}$. From this point the fitting of lines, the determination of the ideal $\Delta \beta_{C / x}$ transformation angle to obtain real minimum horizontal distance and finally the determination of $C P A_{x / x}$ and $t_{C / x}$ (together with $\beta_{C P A / x}$ ) are the same as before. Note that $t_{C / x}$ is the time of closest point in the horizontal plane, in the vertical plane this value can be different.

Turning back to the vertical situation from (12) the remaining equation characterizes the closest point in the vertical plane:

$$
\frac{\bar{y}}{\bar{S}_{y}^{\prime}}=\frac{C P A_{y}^{\prime}}{2}-\frac{V_{y}}{2 R} t_{C P A}
$$

In the above equation $C P A_{y}^{\prime}$ and $t_{C P A}$ are unknown, so the equation can not be solved, despite the constant parameters except for $t_{C P A}$. However, if $t_{C P A}$ is known, this equation can be solved for $C P A_{y}^{\prime}$ which is the relative vertical closest point of approach with respect to the virtual disc. Note that it is only closest if $t_{C P A}$ is determined properly.

If $t_{C / x}$ and so $t_{C P A / x}$ are known from (13) the related $C P A_{y / x}^{\prime}$ value can be determined from (14). This is the relative vertical distance in the point of the minimum relative horizontal one.

Going back to the vertical plane, two cases can arise: one is the case of horizontal flight paths (no crossing in vertical plane $V_{y}=0$ ) when $C P A_{y / y}^{\prime} \neq 0$ will result from (14), and the position of the horizontal minimum distance will give also the 3D minimum distance (see the next section 4 ). In the other case the vertical crossing of the paths means $C P A_{y / y}^{\prime}=0$ and so a special solution of (14) should be obtained. Note that none of the cases require rotation of the body coordinate system in the vertical plane because if one holds the $X, Y$ body plane horizontal $C P A_{y / y}^{\prime} \neq 0$ will be proportional to the altitude difference between the aircraft and $C P A_{y / y}^{\prime}=0$ will mean that the two aircraft are at the same altitude.

$$
\begin{aligned}
& \frac{\bar{y}}{\bar{S}_{y}^{\prime}}=\frac{C P A_{y / y}^{\prime}}{2}-\frac{V_{y}}{2 H} t_{C P A / y}=0-\frac{V_{y}}{2 H}\left(t_{C / y}-t\right)= \\
& =-a_{3} t_{C / y}+a_{3} t=c_{3}+a_{3} t
\end{aligned}
$$

Doing a line fit to the $\left(\frac{\bar{y}}{S_{y}}, t\right)$ data pairs will give $c_{3}$ and $a_{3}$ from which $t_{C / y}$ can be determined:

$$
t_{C / y}=-\frac{c_{3}}{a_{3}}
$$


Going back to the special case when $V_{y}=0$ and $C P A_{y / y}^{\prime} \neq 0$ the equation gives:

$$
\frac{\bar{y}}{\bar{S}_{y}^{\prime}}=\frac{C P A_{y / y}^{\prime}}{2}-0
$$

Regarding the line fit this will give a horizontal line with $t_{C / y}=\infty$ which should be detected upon fitting the line (no intersection with time axis). On the other hand (16) shows that this case it is very easy to obtain $C P A_{y / y}^{\prime}$ from the time average of the $\frac{\bar{y}}{S_{y}^{\prime}}$ values.

Going back to the $C P A_{y / y}^{\prime}=0$ case one will get a $t_{C / y}$ value at which $C P A_{x / y}$ and the related $\Delta \beta_{C / y}$ (and so $\beta_{C P A / y}$ ) values should be determined. This can be done considering the system of equations for the averaged disc size and horizontal data from (13) and (6) ( $\mathrm{s}=\sin , \mathrm{c}=\cos )$.

$$
\left[\begin{array}{c}
\frac{s \beta_{C / y}^{\prime}}{f}-a_{1} \\
c \beta_{C / y}^{\prime}
\end{array}\right]\left[\begin{array}{c}
\frac{C P A_{x / y}}{2} \\
t_{C / y}^{\prime}
\end{array}\right]=\left[\begin{array}{l}
c_{1} \\
c_{2}
\end{array}\right]
$$

as $t_{C / y}$ is known the system can be reformulated having the known values on the right hand side:

$$
\begin{aligned}
& s\left(\beta_{C}+\Delta \beta_{C / y}\right) \frac{C P A_{x / y}}{2 f}=a_{1} t_{C / y}+c_{1}=b_{1} \\
& c\left(\beta_{C}+\Delta \beta_{C / y}\right) \frac{C P A_{x / y}}{2}=a_{2} t_{C / y}+c_{2}=b_{2}
\end{aligned}
$$

In the above system of equations the unknowns are $C P A_{x / y}$ and $\Delta \beta_{C / y}$. Depending on the values of $b_{1}$ and $b_{2}$ there can be different solutions.

1. $b_{1}=0 \& b_{2}=0$ this case $C P A_{x / y}=0$ is the correct solution.

2. $b_{1} \neq 0$ this case the two equations can be divided by each other to remove the unknown $C P A_{x / y}$ value and a solution for $\Delta \beta_{C / y}$ obtained. From this solution $C P A_{x / y}$ can be easily determined.

$$
\begin{aligned}
& c \beta_{C} c \Delta \beta_{C / y}-s \beta_{C} s \Delta \beta_{C / y}= \\
& =\frac{b_{2}}{b_{1} f}\left(s \beta_{C} c \Delta \beta_{C / y}+c \beta_{C} s \Delta \beta_{C / y}\right) \\
& \left(c \beta_{C}-\frac{b_{2}}{b_{1} f} s \beta_{C}\right) c \Delta \beta_{C / y}=\left(s \beta_{C}+\frac{b_{2}}{b_{1} f} c \beta_{C}\right) s \Delta \beta_{C / y} \\
& \tan \Delta \beta_{C / y}=\frac{\left(c \beta_{C}-\frac{b_{2}}{b_{1} f} s \beta_{C}\right)}{\left(s \beta_{C}+\frac{b_{2}}{b_{1} f} c \beta_{C}\right)}
\end{aligned}
$$

3. $b_{2} \neq 0$ this case the two equations can be again divided by each other to remove the unknown $C P A_{x / y}$ value and a solution for $\Delta \beta_{C / y}$ obtained. From this solution $C P A_{x / y}$ can be easily determined. 


$$
\begin{aligned}
& \frac{b_{1} f}{b_{2}}\left(c \beta_{C} c \Delta \beta_{C / y}-s \beta_{C} s \Delta \beta_{C / y}\right)= \\
& =\left(s \beta_{C} c \Delta \beta_{C / y}+c \beta_{C} s \Delta \beta_{C / y}\right) \\
& -\left(c \beta_{C}+\frac{b_{1} f}{b_{2}} s \beta_{C}\right) s \Delta \beta_{C / y}=\left(s \beta_{C}-\frac{b_{1} f}{b_{2}} c \beta_{C}\right) c \Delta \beta_{C / y} \\
& \tan \Delta \beta_{C / y}=\frac{\left(s \beta_{C}-\frac{b_{1} f}{b_{2}} c \beta_{C}\right)}{-\left(c \beta_{C}+\frac{b_{1} f}{b_{2}} s \beta_{C}\right)}
\end{aligned}
$$

Considering both the horizontal and the vertical planes finally eight different parameters can be determined: $t_{C P A / x}\left(t_{C / x}\right), C P A_{x / x}, C P A_{y / x}^{\prime}, \beta_{C P A / x}$ and $t_{C P A / y}\left(t_{C / y}\right), C P A_{x / y}, C P A_{y / y}^{\prime}, \beta_{C P A / y}$. In the special case when $V y=0$ (horizontal aircraft trajectories) the horizontal minimum will give the point of the vertical one also so $t_{C P A / y}=t_{C P A / x}, C P A_{x / y}=C P A_{x / x}, \beta_{C P A / y}=\beta_{C P A / x}$. In the other case $C P A_{y / y}^{\prime}=0$ and the other parameters are usually different and nonzero.

After deriving the minimum distances in the horizontal and vertical planes their relation should be examined compared to the $3 \mathrm{D}$ minimum distance between the aircraft. This relation will be determined in the next section based-on trajectory parameters.

\section{Relation of horizontal, vertical and 3D minimum distances}

The straight intruder flight trajectory relative to the own straight trajectory can be characterized as shown in Fig. 7 .

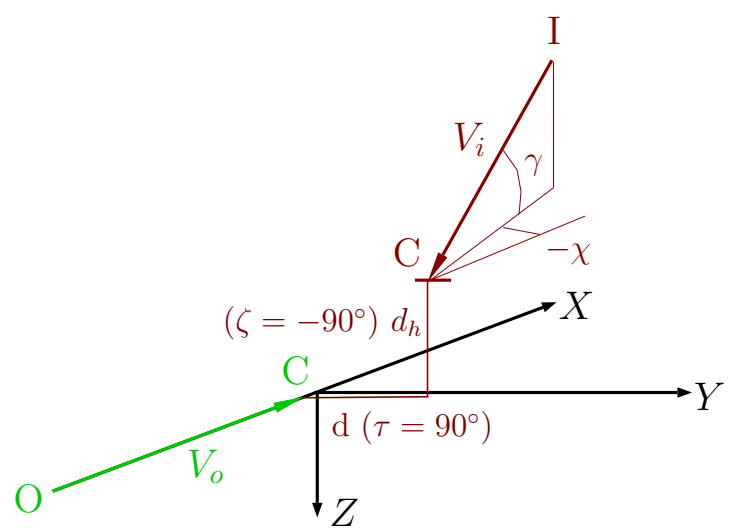

Fig. 7 Own and intruder flight trajectories $\left(V_{o}\right.$ own velocity vector, $V_{i}$ intruder velocity vector, the aircraft are in the $C$ points at the same time

The intruder and own aircraft positions can be formulated from the above figure considering a $t$ time variable decreasing as the aircraft approach the $C$ point $\left(X_{i}=0\right.$ and $X_{o}=0$ in $\left.C\right)$. 


$$
\begin{aligned}
& X_{i}=V_{i} \cos \alpha \cos \beta t, \quad Y_{i}=d+V_{i} \cos \alpha \sin \beta t, \quad Z_{i}=-d_{h}-V_{i} \sin \alpha t \\
& X_{o}=-V_{o} t, \quad Y_{o}=0, \quad Z_{o}=0
\end{aligned}
$$

The $3 \mathrm{D}$, horizontal and vertical distances between the two aircraft result as:

$$
\begin{aligned}
& D_{3 D}=\sqrt{\left(X_{I}-X_{O}\right)^{2}+Y_{I}^{2}+Z_{I}^{2}} \\
& D_{H}=\sqrt{\left(X_{I}-X_{O}\right)^{2}+Y_{I}^{2}} \\
& D_{V}=Z_{I}
\end{aligned}
$$

Taking the square of the distances and calculating the $t$ parameters which give the minimum distances one gets:

$$
\begin{aligned}
& t_{M I N 3 D}=\frac{-d V_{I} \cos \alpha \sin \beta-d_{h} V_{I} \sin \alpha}{V_{I}^{2}+V_{O}^{2}+2 V_{I} V_{O} \cos \alpha \cos \beta} \\
& t_{M I N H}=\frac{-d V_{I} \cos \alpha \sin \beta}{V_{I}^{2} \cos ^{2} \alpha+V_{O}^{2}+2 V_{I} V_{O} \cos \alpha \cos \beta} \\
& t_{M I N V}=\frac{-d_{h} V_{I} \sin \alpha}{\left(V_{I} \sin \alpha\right)^{2}}=\frac{-d_{h}}{V_{I} \sin \alpha}
\end{aligned}
$$

From equation (23) it is apparent that the three minimums will not occur at the same time. The only special case is when $\alpha=0$ which means a horizontal intruder path with $d_{h}$ altitude difference between the aircraft. This case $t_{M I N V}=\infty$ because the paths never intersect in the vertical plane, however $t_{M I N 3 D}=t_{M I N H}$ which means that the $3 \mathrm{D}$ and the horizontal minimum distances coincide. This is obvious if the vertical distance is constant.

Otherwise, the 3D, vertical and horizontal minimums will not coincide. In the previous sections 2 and 3 formuale to obtain the horizontal minimum and the vertical minimum parameters were derived. The question is that if it is possible to somehow obtain the 3D minimum distance from these parameters. Substituting $t_{M I N H}$ and $t_{M I N V}$ into the aircraft positions in (21) one gets two sets of points $((\mathrm{H})$ and $(\mathrm{V})$ respectively):

$$
\begin{aligned}
& X_{i}(H), Y_{i}(H), Z_{i}(H), X_{o}(H) \\
& X_{i}(V), Y_{i}(V), Z_{i}(V), X_{o}(V)
\end{aligned}
$$

In case of straight flight paths interpolation between the vectors connecting the horizontal and vertical own and intruder minimum points and minimization of the resulting $3 \mathrm{D}$ distance can possibly give the coordinates of the $3 \mathrm{D}$ minimum point. The interpolation formulae and the square of the $3 \mathrm{D}$ distance are: 


$$
\begin{aligned}
& X_{M}=X_{i}(H)-X_{o}(H)+t_{M} \frac{X_{i}(V)-X_{o}(V)-\left(X_{i}(H)-X_{o}(H)\right)}{t_{M I N V}-t_{M I N H}}= \\
& =X_{i}(H)-X_{o}(H)+t_{M} \Delta X \\
& Y_{M}=Y_{i}(H)+t_{M} \frac{Y_{i}(V)-Y_{i}(H)}{t_{M I N V}-t_{M I N H}}=Y_{i}(H)+t_{M} \Delta Y \\
& Z_{M}=Z_{i}(H)+t_{M} \frac{Z_{i}(V)-Z_{i}(H)}{t_{M I N V}-t_{M I N H}}=Z_{i}(H)+t_{M} \Delta Z \\
& D^{2}=X_{M}^{2}+Y_{M}^{2}+Z_{M}^{2}
\end{aligned}
$$

The further calculations were done in Matlab ${ }^{\circledR}$ Symbolic Toolbox ${ }^{\circledR}$. Minimization of the square of $3 \mathrm{D}$ distance $D^{2}$ gave $t_{M}=t_{M I N 3 D}$ so the time of the real 3D minimum distance and also by substitution the coordinates of the 3D minimum distance $X_{i}(3 D), Y_{i}(3 D), Z_{i}(3 D), X_{o}(3 D)$ result. This means that from the horizontal and vertical minimum points the $3 \mathrm{D}$ minimum can be obtained by simple interpolation.

\section{An interpolation method to estimate 3D TTCPA and CPA values}

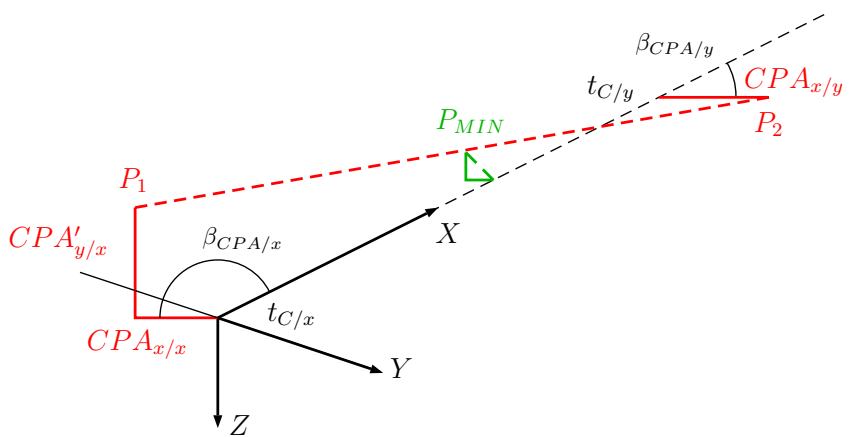

Fig. 8 Special points along the 3D aircraft trajectories

Based-on the result of the previous section interpolation should give $t_{C P A}, C P A_{x}$ with $\beta_{C P A}$ and $C P A_{y}^{\prime}$ of the $3 \mathrm{D}$ minimum point between the two aircraft in S\&A.

Summarizing the previous developments one can determine two special points between the aircraft trajectories. The first can be the point where the horizontal distance is minimum and the related vertical distance can be also determined. Important parameters in this point can be denoted as:

$$
C P A_{x / x}, \beta_{C P A / x}, C P A_{y / x}^{\prime}, t_{C / x}
$$

In the special case when the vertical distance between the aircraft is constant, this first point gives also the 3D minimum distance between the two aircraft. In 
the case, when the vertical trajectories cross each other the second point is this crossing with $C P A_{y / y}^{\prime}=0$. Important parameters in this point can be denoted as:

$$
C P A_{x / y}, \beta_{C P A / y}, C P A_{y / y}^{\prime}, t_{C / y}
$$

Fig. 8 shows the two points as $P_{1}$ and $P_{2}$.

The coordinates of points $P_{1}$ and $P_{2}$ in the body coordinate system (X, Y, Z in Fig. 8) so result as:

$$
\begin{aligned}
& P_{1}: \\
& X_{1}=c \beta_{C P A / x} C P A_{x / x} \\
& Y_{1}=s \beta_{C P A / x} C P A_{x / x} \\
& Z_{1}=C P A_{y / x}^{\prime} \\
& P_{2}: \\
& X_{2}=c \beta_{C P A / y} C P A_{x / y} \\
& Y_{2}=s \beta_{C P A / y} C P A_{x / y} \\
& Z_{2}=0
\end{aligned}
$$

An interpolated point between $P_{1}$ and $P_{2}$ can be represented as follows. Note that the given representation with the $t_{M}$ parameter makes extrapolation before $P_{1}$ or after $P_{2}$ also possible.

$$
\begin{aligned}
& X_{M}=X_{1}+t_{M} \frac{X_{2}-X_{1}}{t_{C / y}-t_{C / x}}=X_{1}+t_{M} \Delta X \\
& Y_{M}=Y_{1}+t_{M} \frac{Y_{2}-Y_{1}}{t_{C / y}-t_{C / x}}=Y_{1}+t_{M} \Delta Y \\
& Z_{M}=Z_{1}+t_{M} \frac{Z_{2}-Z_{1}}{t_{C / y}-t_{C / x}}=Z_{1}+t_{M} \Delta Z
\end{aligned}
$$

As the points are represented relative to the own aircraft body coordinate system, the distance between own aircraft and intruder can be represented as the absolute value of this vector at every point. Taking the square of the distance the $t_{M}$ parameter giving the minimum distance can be calculated taking minimization with respect to it:

$$
\begin{aligned}
D= & \sqrt{X_{M}^{2}+Y_{M}^{2}+Z_{M}^{2}} \rightarrow \\
D^{2}= & X_{M}^{2}+Y_{M}^{2}+Z_{M}^{2} \\
D^{2}= & X_{1}^{2}+Y_{1}^{2}+Z_{1}^{2}+ \\
& +2\left(X_{1} \Delta X+Y_{1} \Delta Y+Z_{1} \Delta Z\right) t_{M}+ \\
& +\left(\Delta X^{2}+\Delta Y^{2}+\Delta Z^{2}\right) t_{M}^{2} \\
\frac{\partial D^{2}}{\partial t_{M}}= & =2\left(X_{1} \Delta X+Y_{1} \Delta Y+Z_{1} \Delta Z\right)+ \\
& +2\left(\Delta X^{2}+\Delta Y^{2}+\Delta Z^{2}\right) t_{M} \rightarrow \\
t_{M}= & -\frac{X_{1} \Delta X+Y_{1} \Delta Y+Z_{1} \Delta Z}{\Delta X^{2}+\Delta Y^{2}+\Delta Z^{2}}
\end{aligned}
$$


Considering this result the time of minimum 3D distance and the related relative CPA values can be determined as follows:

$$
\begin{aligned}
& t_{C M I N}=t_{C / x}+t_{M} \\
& t_{C P A M I N}=t_{C M I N}-t \\
& C P A_{y M I N}^{\prime}=Z_{M} \\
& C P A_{x M I N}=\sqrt{X_{M}^{2}+Y_{M}^{2}} \\
& \tan \beta_{C P A M I N}=\frac{Y_{M}}{X_{M}}
\end{aligned}
$$

Finally, the vertical CPA related to the $H$ vertical size can be calculated considering the ratio between horizontal and vertical sizes:

$$
k=\frac{H}{R}
$$

Taking the time averaged size ratio $\bar{k}$ one can transform $C P A_{y}^{\prime}$ related to $R$ to $C P A_{y}$ related to $H$ :

$$
C P A_{y M I N}=\frac{Y_{a}}{H}=\frac{1}{\bar{k}} C P A_{y M I N}^{\prime}
$$

The only question is the estimation of the size ratio.

\subsection{Estimation of the size ratio}

As discussed before related to Fig. 6 the size of the intruder image depends on the view angle of the intruder both in horizontal and vertical plane. That's why before calculating the size ratio the measured $S_{x}$ and $S_{y}$ sizes should be transformed to $\beta=0$ and $\alpha=0$ central positions following the steps discussed in section 3 but substituting 0 in place of the second view angle $(\alpha)$ in the transformation (10). The $k$ size ratio can be calculated as the ratio of the obtained $S_{y 0}$ and $S_{x 0}$ transformed sizes:

$$
k=\frac{S_{y 0}}{S_{x 0}}
$$

After developing a method to determine the 3D minimum distance between two aircraft in a close encounter scenario it should be tested in detail running simulations with many trajectory, intruder size and velocity parameters. This is presented in the next section.

\section{SIL test environment and Monte-Carlo test results}

A SIL test environment is built in Matlab ${ }^{\circledR}$ to test the developed S\&A estimation algorithm. In this environment the intruder aircraft is represented by a point set constructed from the vertices of the rectangles shown in Fig. 9. 


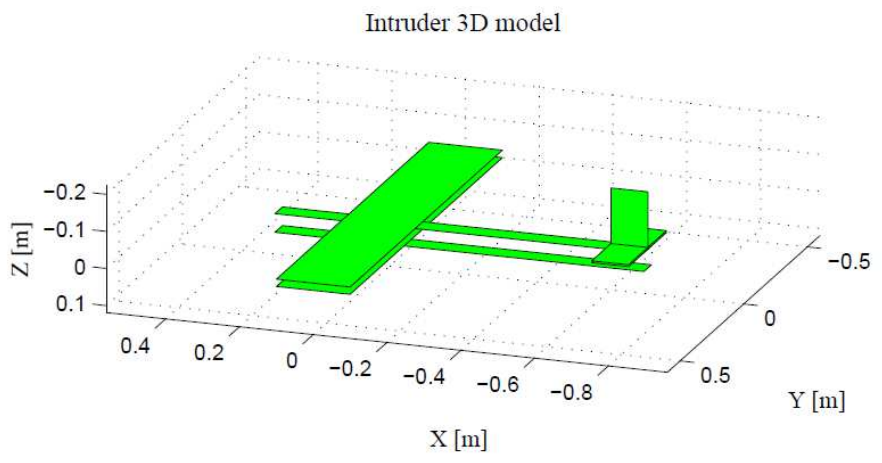

Fig. 9 Intruder 3D model (only the vertices are considered)

This geometric model is scaled to the actual size and projected using pinhole camera model in the six camera system with $360^{\circ}$ horizontal field of view presented in [3] and [2]. Pixelization errors are considered so $\left\{x, y, S_{x}, S_{y}\right\} \in \mathbb{Z}$. During the processing of the pixelized image data ego motion compensation (proposed in [7]) is also applied.

The tipical intruder sizes and velocities are obtained from the size-speed characteristic in Fig. 16 and summarized in Table 1.

Table 1 Intruder sizes and velocities

\begin{tabular}{llllll}
\hline Size $(\mathrm{R})[\mathrm{m}]$ & 3.5 & 10 & 20 & 40 & 60 \\
\hline Height $(\mathrm{H})[\mathrm{m}]$ & 0.7 & 3 & 5.9 & 11.7 & 17.4 \\
\hline$V_{\min }[\mathrm{m} / \mathrm{s}]$ & 10 & 39 & 52 & 133 & 205 \\
$V_{\operatorname{mean}}[\mathrm{m} / \mathrm{s}]$ & 25 & 72 & 145 & 222 & 241 \\
$V_{\max }[\mathrm{m} / \mathrm{s}]$ & 40 & 147 & 256 & 265 & 257 \\
\hline
\end{tabular}

The intruder trajectories relative to the own can be characterized by $\alpha, \beta, \tau$, $\zeta, H C P A$ and $V C P A$ as presented in Fig. 7. In the figure $d=\sin (\tau) H C P A \cdot R$ and $d_{h}=\sin (\zeta) V C P A \cdot H$.

In the Monte-Carlo test campaign the considered HCPA and VCPA sets are $H C P A=[0,5,10]$ and $V C P A=[0,5,10]$ respectively. The $\alpha, \beta, \tau, \zeta$ parameters are combined to cover every possible situation regarding the directions between own aircraft and intruder. The own aircraft is assumed to fly straight and level with $V_{o}=20 \mathrm{~m} / \mathrm{s}$. Intruder can come from below / over, from left / right and arrive below / over and left / right. Table 2 summarizes the considered parameter sets $(\mathrm{B}=$ below, $\mathrm{O}=$ over, $\mathrm{L}=$ left, $\mathrm{R}=$ right $)$. 
Table 2 Intruder trajectory parameter sets

\begin{tabular}{lllllllll}
\hline $\mathrm{NR}$ & 1 & 2 & 3 & 4 & 5 & 6 & 7 & 8 \\
\hline$\beta[\mathrm{deg}]$ & 0 & 45 & 90 & 135 & 180 & 225 & 270 & 315 \\
\hline$\alpha[\mathrm{deg}]$ & 5 & 5 & 0 & -5 & -5 & -5 & 0 & 5 \\
\hline$\tau[\mathrm{deg}]$ & 90 & 90 & 90 & 90 & 90 & -90 & -90 & -90 \\
\hline$\zeta[\mathrm{deg}]$ & 90 & -90 & 90 & -90 & 90 & -90 & 90 & -90 \\
\hline $\mathrm{FROM}$ & $-/ \mathrm{O}$ & $\mathrm{R} / \mathrm{O}$ & $\mathrm{R} /-$ & $\mathrm{R} / \mathrm{B}$ & $-/ \mathrm{B}$ & $\mathrm{L} / \mathrm{B}$ & $\mathrm{L} /-$ & $\mathrm{L} / \mathrm{O}$ \\
\hline ARRIVE & $\mathrm{R} / \mathrm{B}$ & $\mathrm{R} / \mathrm{O}$ & $\mathrm{R} / \mathrm{B}$ & $\mathrm{R} / \mathrm{O}$ & $\mathrm{R} / \mathrm{B}$ & $\mathrm{L} / \mathrm{O}$ & $\mathrm{L} / \mathrm{B}$ & $\mathrm{L} / \mathrm{O}$ \\
\hline
\end{tabular}

The own aircraft is simulated considering the dynamics of its autopilot, the intruder is simulated as a moving point mass along a straight line. All possible combinations of intruder $R$ sizes and velocities from Table 1 (except for $V_{i}=10 \mathrm{~m} / \mathrm{s}$ as this is below $V_{o}$ and so the intruder coming from back can not reach the own aicraft) with all possible combinations of HCPA, VCPA and the intruder trajectory parameter sets from Table 2 were simulated (the overall number of simulated cases is 1008).

\subsection{Results of simulation campaign}

To decide about the possibility of collision a threshold for $t_{C P A}$ and the $C P A$ values should be provided. The $t_{C P A}$ threshold should be selected based-on the maneuvering capability of the own aircraft. Now it was selected to be 3 seconds (s) which could be enough to execute avoidance. If the estimated $t_{C P A M I N}$ value is below $3 s$ then the $C P A$ values should be checked to decide about collision. The decision threshold for $\left|C P A_{x M I N}\right|$ and $\left|C P A_{y M I N}\right|$ was set to be 7 (consider HCPA and VCPA 0 and 5 cases as collision and 10 cases as non collision). Above this value there is no collision, below it there is. Considering the horizontal and vertical data if either $\left|C P A_{x M I N}\right|$ or $\left|C P A_{y M I N}\right|$ is above the threshold there won't be a collision.

The parameters used in the evaluation are as follows:

- The real $t_{C P A}$ value from aircraft trajectory data when $t_{C P A M I N}<3$ and decision is done.

- The real $C P A_{x}, C P A_{y}^{\prime}$ and $C P A_{y}$ values obtained from trajectory data and the actual sizes of the intruder $(R$ and $H)$.

- The real size ratio $k=H / R$ of the intruder.

- The real direction $\beta_{C P A}$ of the closest point.

Considering the decisions the success rates are determined based-on the values of the real $C P A_{x}$ and $C P A_{y}$ parameters when the decision is done. The real situation is considered as a collision if $\left|C P A_{x}\right|<7$ and $\left|C P A_{y}\right|<7$. Table 3 shows the number of tested scenarios (NR), the success rates (SR), the false alarm (FA, when collision danger is detected while there is no danger) and missed detection (MD, when no danger detected while there is one) rates for each intruder size.

The table shows that the decision success rate is above $80 \%$ in all cases (above $85 \%$ in most of the cases). According to [18] the overall S\&A system effectiveness 
requirement ranges from $30.9 \%$ to $68.9 \%$ for the considered own aircraft categories (see section A). Our results satisfy this very well if one considers also that the false alarm rates are high $(7-14 \%)$ but the missed detection rates are very low (below $3 \%$ ). This means that the proposed decision method is a bit conservative and so it rather does an avoidance which is not required than collide in the intruder which increases overall system safety.

The details of the obtained estimated and real parameters are shown in Fig.s 10 to 15. In all of the figures the coloured three groups of objects mean the test results for one aircraft size with $H C P A=[0,5,10]$ values. So index 1 is $R=3.5 \mathrm{~m}$ and $H C P A=0$, index 2 is $R=3.5 \mathrm{~m}$ and $H C P A=5$, index 3 is $R=3.5 \mathrm{~m}$ and $H C P A=10$, index 4 is $R=10 \mathrm{~m}$ and $H C P A=0$ etc.

Table 3 SIL test results

\begin{tabular}{llllll}
\hline Size (R) [m] & 3.5 & 10 & 20 & 40 & 60 \\
\hline NR & 144 & 216 & 216 & 216 & 216 \\
\hline SR [\%] & 88.2 & 92.6 & 86.6 & 85.2 & 83.3 \\
FA [\%] & 11.8 & 7.4 & 11.6 & 12 & 13.9 \\
MD [\%] & 0 & 0 & 1.8 & 2.8 & 2.8 \\
\hline
\end{tabular}

Fig. 10 shows the real $t_{C P A}$ values when $t_{C P A M I N}<3 \mathrm{~s}$ and the decision is done. In most of the cases the real values are above 2.5 seconds which means that the own aircraft has this time to make the avoidance after decision. Compared to the 3 seconds threshold this is a really good accuracy. Only 5 values are below $2.5 \mathrm{~s}$ and no value is under $2.25 \mathrm{~s}$. The 0 value means that the intruder was in the deadzone in the overlapping region of two cameras when the decision would be done. In this overlapping zone the estmation of intruder parameters is impossible because none of the cameras tracks fully the intruder.

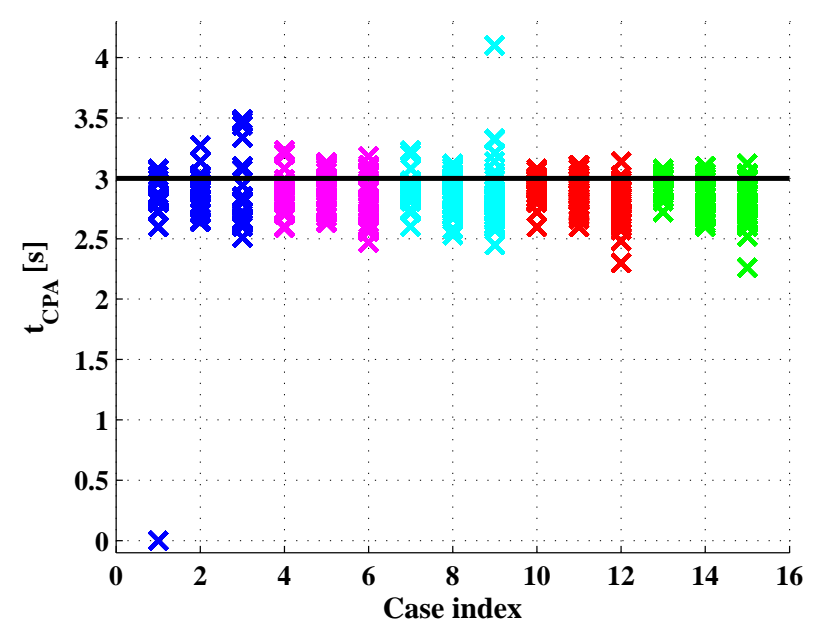

Fig. 10 Real $t_{C P A}$ at the time of decision 


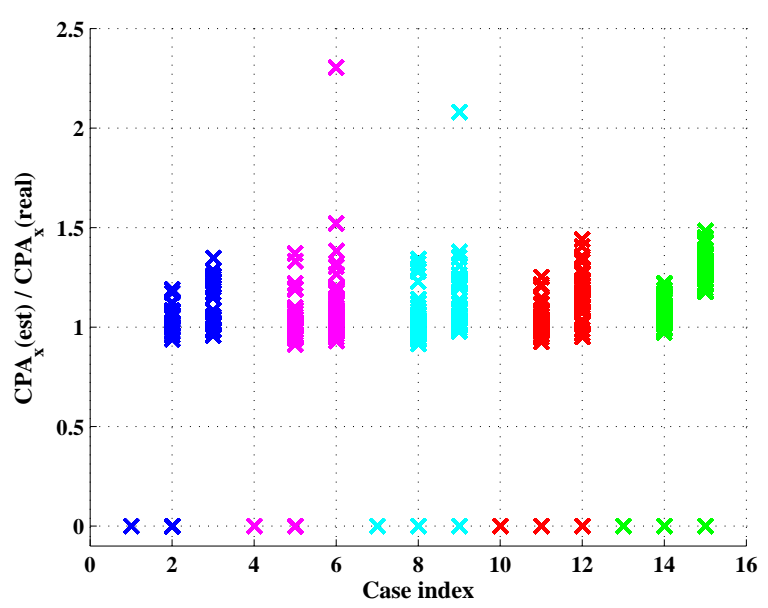

Fig. 11 Ratio of estimated and real $C P A_{x}$ values

Fig. 11 shows the ratios of the estimated $\left|C P A_{x M I N}\right|$ and the real $\left|C P A_{x}\right|$ values. The zero values represent the cases when both $\left|C P A_{x M I N}\right|$ and $\left|C P A_{x}\right|$ are below 1 and this would give a very uncertain ratio. Most of the values are between 0.9 and 1.5 which means that the $C P A_{x}$ horizontal distance is usually overestimated. That's why the threshold 7 was selected to consider $C P A=5$ as a collision. This means that overestimation with a ratio of 1.4 will still give collision. Only four ratios are above 2 in case of $\mathrm{R}=10 \mathrm{~m}$ and $\mathrm{R}=20 \mathrm{~m}$ intruders with $\mathrm{HCPA}=10$ and $\mathrm{VCPA}=10$. In these situations there is a camera change (intruder image moves from one camera to another) just before the decision and the transient in the estimates causes this error.

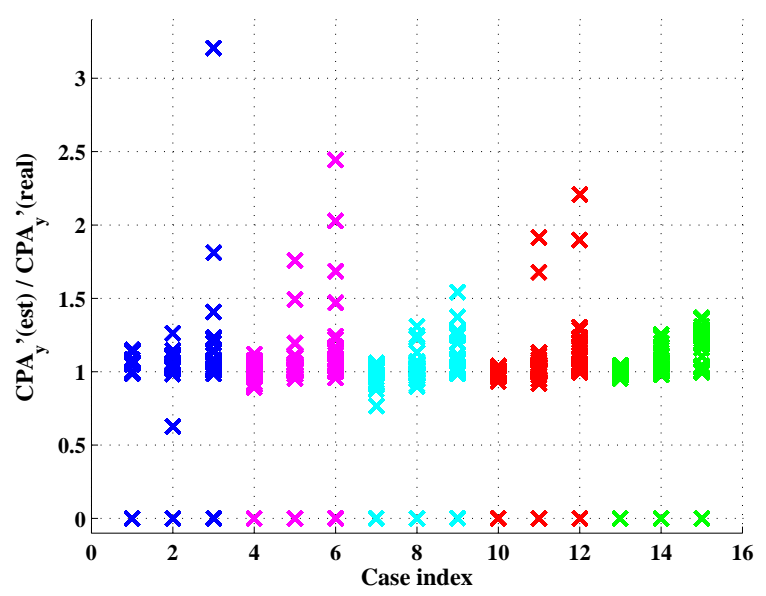

Fig. 12 Ratio of estimated and real $C P A_{y}^{\prime}$ values 
Fig. 12 shows the ratios of the estimated $\left|C P A_{y M I N}^{\prime}\right|$ and the real $\left|C P A_{y}^{\prime}\right|$ values so the CPA-s relative to the virtual vertical size of the intruder. The zero values again represent the cases when both $\left|C P A_{y M I N}^{\prime}\right|$ and $\left|C P A_{y}^{\prime}\right|$ are below 1 and this would give a very uncertain ratio. Most of the values are again between 0.9 and 1.5 but there are more overestimated value above 1.5. This means that this is not a good basis for decision because can cause more missed detections. In the actual simulations $\left|C P A_{y M I N}\right|$ is used in the vertical decision which is underestimated and so gives false alarms, but increases safety.

Fig. 13 shows the ratios of the estimated $\left|C P A_{y M I N}\right|$ and the real $\left|C P A_{y}\right|$ values so the CPA-s relative to the real vertical size of the intruder. The zero values again represent the cases when both $\left|C P A_{y M I N}\right|$ and $\left|C P A_{y}\right|$ are below 1 and this would give a very uncertain ratio. For this parameter most of the ratios are between 0.4 and 1.2 which shows the underestimation of the parameter. This gives conservative estimates and so false alarms but this is better than missed detections. Neither of the values is above 2. Underestimation is mainly caused by the overestimation of the size ratio $k$ see Fig. 15 .

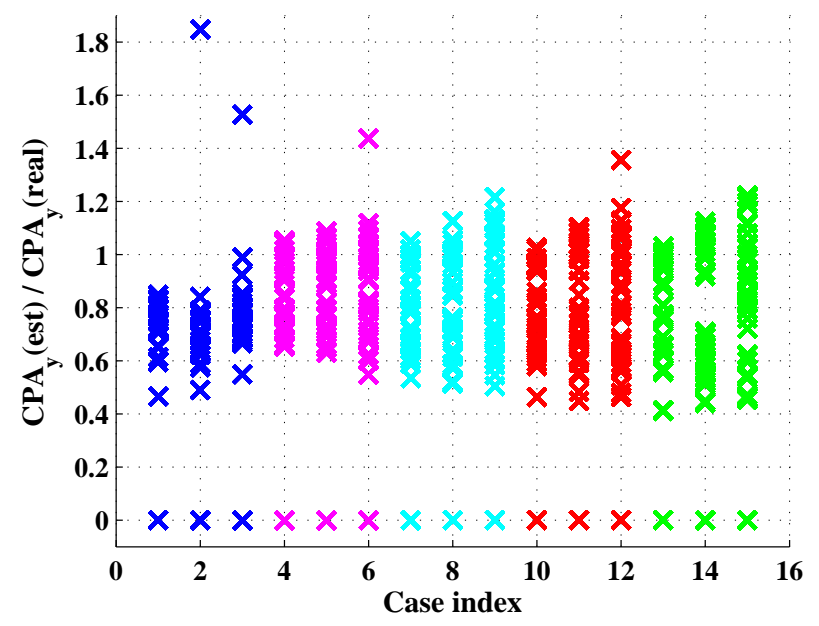

Fig. 13 Ratio of estimated and real $C P A_{y}$ values

Fig. 14 shows the error $\Delta \beta_{C P A}$ between the estimated and real horizontal $C P A_{x}$ direction values $\beta_{C P A}$ visualized as unit vectors with $\Delta \beta_{C P A}$ angles relative to the vertical direction. The figure shows that the precision of the direction estimation is good, in most of the cases the error is below $10^{\circ}$. Only the HCPA $=0$ columns show larger deviations because for an almost zero $C P A_{x}$ value the estimate of its direction is very uncertain. Here the indices ranging from 1 to 15 in the previous figures are multiplied by 3 so they range from 3 to 45 but they represent the same sizes and HCPA values. 


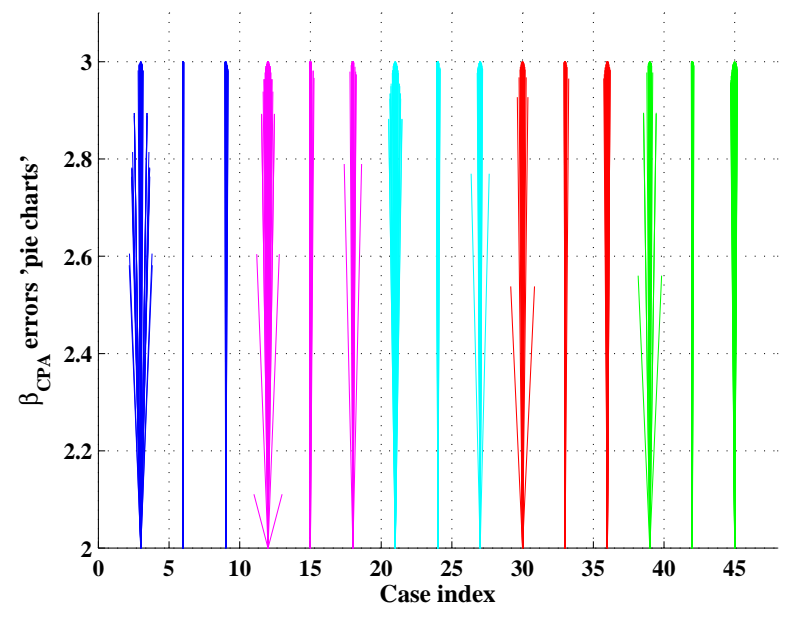

Fig. 14 Representation of $\beta_{C P A}$ estimation errors

Finally, Fig. 15 shows the estimated size ratios of the intruder aircraft and the real ratios (as circles) also. Most of the ratios are overestimated mainly because the intruder is not seen fully frontally and so the fuselage can give projected vertical points which virtually increase the height of the intruder image. In the future a more precise ratio estimation method should be developed if possible to decrease also the underestimation of $C P A_{y}$.

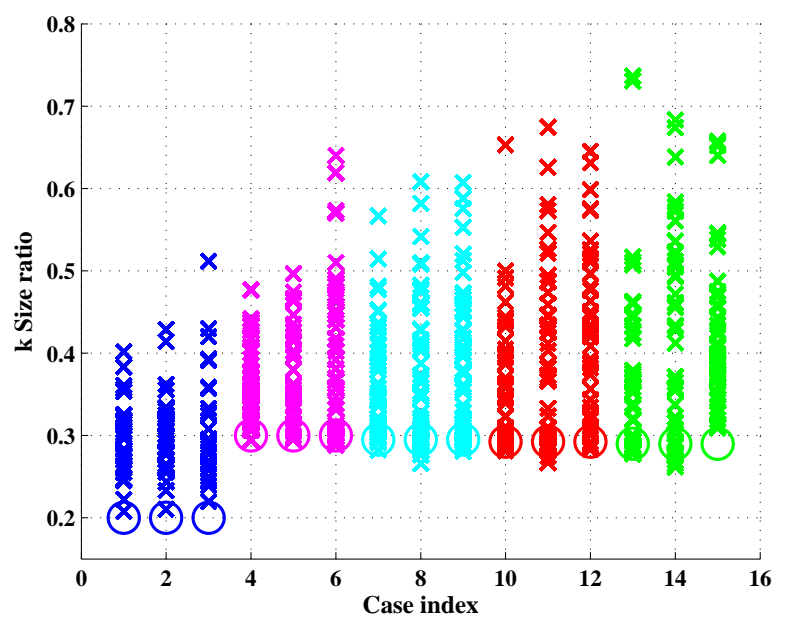

Fig. 15 Real and estimated intruder size ratios 


\section{Conclusion}

This paper deals with aircraft monocular image-based sense and avoid which is an actual and widely researched topic. The main difficulty of the use of monocular images is the loss of 3D information and the resulting scale ambiguity in intruder aircraft size and distance. Usually only the intruder image size and position information in the image plane is available. The goal of the authors was to extract the utmost information from this data considering consecutive image frames assuming constant aircraft speeds and straight trajectories.

The first part summarizes the author's previous results focusing on the collision situation in the horizontal plane considering $360^{\circ}$ field of view of a muliple camera vision system. The intruder aircraft geometry is modelled as a disc because the wingspan/length ratio of the aircraft is usually around 1 as shown in the appendix. Finally, it is possible to estimate the time to closest point of approach, the relative distance (CPA) and its direction at this time. This is enough to make a collision decision regarding the horizontal situation.

However, the vertical closeness of the aircraft is equally important. Because the disc model can not be immediately applied in the vertical plane as the height / horizontal size ratio is far from 1 a virtual extension of the vertical size to the size of the horizontal disc is proposed. Then the horizontal formulae can be applied also in the vertical plane. Combining all formulae its finally possible to determine the time to closest point of approach, the relative distance (CPA) and its direction both in the horizontal and vertical planes.

Usually, the horizontal and vertical closest points do not coincide as is pointed out based-on trajectory geometries. However, interpolation between the two points can give the global 3D minimum closest point and the related time. This is first pointed out based-on trajectory geometries, then the method is applied on the estimated, image-based horizontal and vertical parameters. Finally, the 3D closest point position is characterized by its TTCPA, horizontal $C P A_{x}$ and vertical $C P A_{y}$ relative distances and the direction of the horizontal component. Because of the scale ambiguity only the distances relative to the intruder horizontal and vertical size can be determined. First they are all related to the horizontal size of the intruder (because of the virtual vertical disc), then the vertical distance is transformed by the intruder size ratio to make it relative to the vertical size. These relative sizes are enough to select a threshold and make a decision if the intruder will be close in the three dimensional space or not.

Future developments should include real flight testing (flight test videos of the horizontal part of the decision can be seen on our Youtube / AeroGNC channel), derivation of improved intruder size ratio formulae as the current one is very inaccurate, the proposal of an avoidance strategy based-on the estimated 3D situation and extension of the developments towards non straight flight trajectories.

\section{A Intruder characterization based-on real aircraft data}

The possible airspace categories where the proposed S\&A system can be used are selected based on [18] which makes an important effort to set S\&A system effectiveness standards considering different class of UAVs and airspaces. The targeted airspaces by current development are Class D/E and $\mathrm{G}$ which does not require on-board transponder or ATC link. The targeted own aircraft categories are Group 1 to 4 (micro to tactical). Considering the possible threats, 
in Class D/E airspaces the intruder aircrafts can range from micro UAVs through general aviation (GA) aircraft until large airliners / transporters on their approach to airports. So, these types should be characterized. [18] characterizes A/Cs based-on their weight and speed, however from a vision sensor point of view it is better to use size and speed.

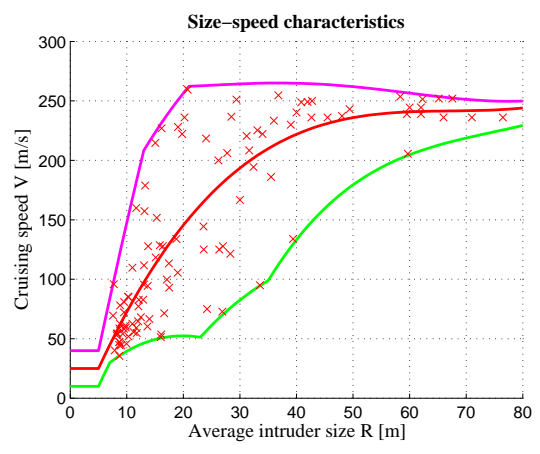

Fig. 16 Possible intruder aircraft sizes and related cruise speeds

Wingspan (b), fuselage length $(L)$, height $(H)$ and cruise speed characteristics were collected from [1] ranging from CAP-10 to Airbus A380 and AN-225 including also helicopters. Three characteristic diagrams were obtained. The first is the horizontal size-speed diagram in Fig. 16 which shows the cruise speeds of the aerial vehicles against their average (mean of wingspan (rotor diameter) and length) horizontal size (R). Minimum, mean and maximum size-cruise speed curves are fitted to the data which can be used in the generation of intruder aircraft data in Monte-Carlo tests.

The second is the histogram for the wingspan (b) / length (L) ratios in Fig. 17. This shows that most of the ratios are close to 1 and so the horizontal disc geometrical model presented in section 2 can be valid.

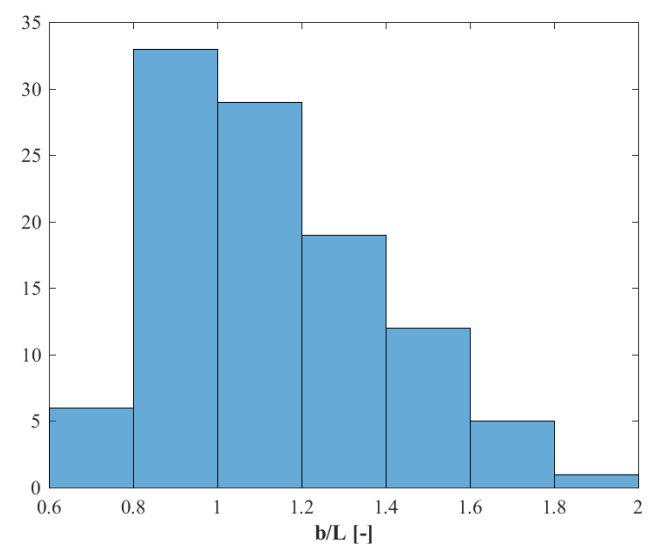

Fig. 17 Histogram of wingspan (b) / length (L) ratios

The third is the histogram for the height $(H) /$ average size $(R)$ ratios in Fig. 18. This shows that height / horizontal size can range from 0.2 to 0.4 and most of the data is around 0.3 . 


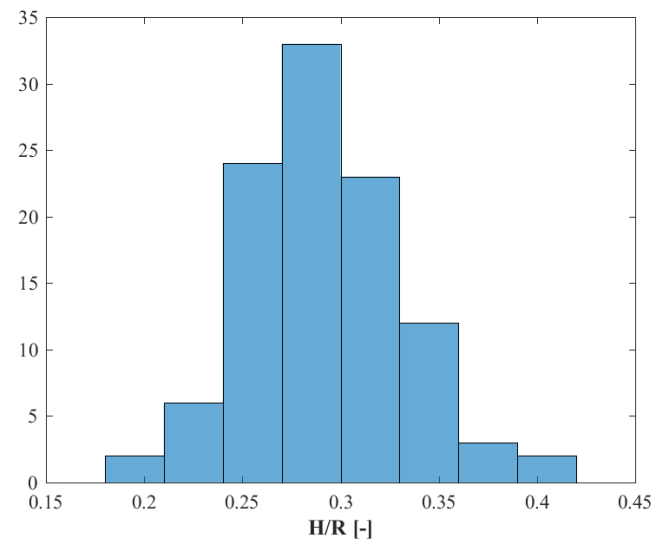

Fig. 18 Histogram of height $(\mathrm{H}) /$ average size $(\mathrm{R})$ ratios

\section{References}

1. airliners.net: aircraft-data (2015). URL http://www.airliners.net/aircraft-data/

2. Bauer, P., Hiba, A.: Vision Only Collision Detection with Omnidirectional Multi-Camera System. In: in Proc. of the 20th World Congress of the International Federation of Automatic Control, pp. 15,780-15,785. IFAC, Toulouse, France (2017)

3. Bauer, P., Hiba, A., Bokor, J.: Monocular Image-based Intruder Direction Estimation at Closest Point of Approach. In: in Proc. of the International Conference on Unmanned Aircraft Systems (ICUAS) 2017, pp. 1108-1117. ICUAS Association, Miami, FL, USA (2017)

4. Bauer, P., Hiba, A., Vanek, B., Zarandy, A., Bokor, J.: Monocular Image-based Time to Collision and Closest Point of Approach Estimation. In: In proceedings of 24th Mediterranean Conference on Control and Automation (MED'16). Athens, Greece (2016)

5. Bauer, P., Vanek, B., Pni, T., Zsedrovits, T., Pencz, B., kos Zarndy, Bokor, J.: Aircraft Trajectory Tracking with Large Sideslip Angles for Sense and Avoid Intruder State Estimation. In: In proceedings of 22nd Mediterranean Conference on Control and Automation (MED'14). Palermo, Italy (2014)

6. Byrne, J., Taylor, C.J.: Expansion Segmentation for Visual Collision Detection and Estimation. In: In Proc. of IEEE International Conference on Robotics and Automation (2009)

7. Degen, S.: Reactive Image-based Collision Avoidance System for Unmanned Aircraft Systems. Master's thesis, Australian Research Centre for Aerospace Automation (2011)

8. Dempsey, M.: U.s. army unmanned aircraft systems roadmap 2010-2035. Tech. rep., U.S. Army UAS Center of Excellence (2010)

9. EU: Roadmap for the integration of civil Remotely-Piloted Aircraft Systems into the European Aviation System. Tech. rep., European RPAS Steering Group (2013)

10. FAA: Federal Aviation Regulation 14 CFR Part 91. Federal Aviation Administration (FAA) (2016)

11. Fasano, G., Accardo, D., Forlenza, L., Moccia, A., Rispoli, A.: A multi-sensor obstacle detection and tracking system for autonomousuav sense and avoid. In: $X X$ Congresso Nazionale AIDAA, Milano (2009)

12. Fasano, G., Accardo, D., Tirri, A.E., Moccia, A.: Feature article: Experimental analysis of onboard non-cooperative sense and avoid solutions based on radar, optical sensors, and data fusion. IEEE Aerospace and Electronic Systems Magazine 31(7), 6-14 (2016). DOI 10.1109/MAES.2016.150164

13. Forlenza, L.: Vision based strategies for implementing Sense and Avoid capabilities onboard Unmanned Aerial Systems. Ph.D. thesis, UNIVERSITÁ DEGLI STUDI DI NAPOLI FEDERICO II (2012) 
14. Frew, E.W.: Observer Trajectory Generation for Target-Motion Estimation Using Monocular Vision. Ph.D. thesis, Stanford University (2003)

15. Hutchings, T., Jeffryes, S., Farmer, S.J.: Architecting uav sense \& avoid systems. In: Proc. Institution of Engineering and Technology Conf. Autonomous Systems, pp. 1-8 (2007)

16. Lyu, Y., Pan, Q., Zhao, C., Zhang, Y., Hu, J.: Feature article: Vision-based UAV collision avoidance with 2D dynamic safety envelope. IEEE Aerospace and Electronic Systems Magazine 31(7), 16-26 (2016). DOI 10.1109/MAES.2016.150155

17. Mejias, L., McFadyen, A., Ford, J.J.: Feature article: Sense and avoid technology developments at Queensland University of Technology. IEEE Aerospace and Electronic Systems Magazine 31(7), 28-37 (2016). DOI 10.1109/MAES.2016.150157

18. Melnyk, R., Schrage, D., Volovoi, V., Jimenez, H.: Sense and Avoid Requirements for Unmanned Aircraft Systems Using a Target Level of Safety Approach. Risk Analysis 34(10), 1894-1906 (2014)

19. Meyer, F., Bouthemy, P.: Estimation of time-to-collision maps from first order motion models and normal flows. In: In Proc. of 11th IAPR International Conference on Pattern Recognition (1992)

20. Mori, T., Scherer, S.: First Results in Detecting and Avoiding Frontal Obstacles from a Monocular Camera for Micro Unmanned Aerial Vehicles. In: International Conference on Robotics and Automation (2013)

21. Nussberger, A., Grabner, H., Gool, L.V.: Feature article: Robust Aerial Object Tracking from an Airborne platform. IEEE Aerospace and Electronic Systems Magazine 31(7), 38-46 (2016). DOI 10.1109/MAES.2016.150126

22. O., S., W.-Z., C., M., R.M.V.: Passive ranging for uav sense and avoid applications. In: In fotech Aerospace (2005)

23. Salazar, L.R., Sabatini, R., Ramasamy, S., Gardi, A.: A Novel System for Non-Cooperative UAV Sense-And-Avoid. In: In Proceedings of European Navigation Conference 2013 (ENC 2013) (2013)

24. Schaub, A., Burschka, D.: Spatio-Temporal Prediction of Collision Candidates for Static and Dynamic Objects in Monocular Image Sequences. In: In Proc. of IEEE Intelligent Vehicles Symposium (IV 2013) (2013)

25. Speijker, L., Verstraeten, J., Kranenburg, C., van der Geest, P.: Scoping Improvements to 'See And Avoid' for General Aviation (SISA). Tech. rep., European Aviation Safety Agency (EASA) (2012)

26. Vanek, B., Peni, T., Zarandy, A., Bokor, J., Zsedrovits, T., Roska, T.: Performance Characteristics of a Complete Vision Only Sense and Avoid System. In: in Proceedings of AIAA GNC 2012 (Guidance, Navigation and Control Conference), AIAA 2012-4703, pp. 1-15. Minneapolis, Minnesota (2012)

27. Watanabe, Y.: Stochastically Optimized Monocular Vision-based Navigation and Guidance. Ph.D. thesis, Georgia Institute of Technology (2008)

28. Zarandy, A., Nagy, Z., Vanek, B., Zsedrovits, T., Kiss, A., Nemeth, M.: A Five-Camera Vision System for UAV Visual Attitude Calculation and Collision Warning. In: Computer Vision Systems, Lecture Notes in Computer Science, pp. 11-20. Saint Petersburg, Russia (2013)

29. Zsedrovits, T., Bauer, P., Pencz, B.J.M., Hiba, A., Gőzse, I., Kisantal, M., Németh, M., Nagy, Z., Vanek, B., Zarándy, A., Bokor, J.: Onboard Visual Sense and Avoid System for Small Aircraft. IEEE A\&E SYSTEMS MAGAZINE 31, 18-27 (2016)

30. Zsedrovits, T., Zarandy, A., Vanek, B., Peni, T., Bokor, J., Roska, T.: Collision avoidance for UAV using visual detection. In: in Proceedings of IEEE ISCAS 2011 (International Symposium on Circuits and Systems), pp. 2173-2176. IEEE, Rio de Janeiro (2011)

\section{B Author short bios}

Peter Bauer received his MSc and PhD in Mechanical Engineering (Aerospace Engineering) in 2005 and 2013, respectively, at Budapest University of Technology and Economics, Budapest, Hungary. He is a Senior Research Fellow at the Institute for Computer Science and Control, Hungarian Academy of Sciences, Budapest, Hungary. His research fields cover state and parameter estimation, tracking control design, aircraft autopilot and sense and avoid system development. He also designs and coordinates flight test experiences. 
Antal Hiba received his MSc in electronic and computer engineering and graduate to a computer engineer specialized in electronic business in 2010 and 2012 at Pazmany Peter Catholic University (PPCU), Budapest, Hungary. He received the Ph.D. in Information Technology at PPCU in 2016. Currently, he is a researcher at the Institute for Computer Science and Control of the Hungarian Academy of Sciences (MTA-SZTAKI). His research interests are real-time embedded visual systems and high performance computing with dataflow architectures.

Jozsef Bokor received Dr.Univ. and $\mathrm{PhD}$ degrees in electrical engineering from the $\mathrm{Bu}-$ dapest University of Technology and Economics (BUTE), in 1977 and 1983, respectively, and a DSc degree in control from the Hungarian Academy of Sciences (HAS) in 1990. He was elected a corresponding member and later a full member of the HAS in 1999 and 2002, respectively. Currently, he is a research director and the head of the Systems and Control Laboratory of the Institute for Computer Science and Control, HAS. He is also a professor at the Department of Control and Transport Automation, Faculty of Transportation Engineering, BUTE. He has held visiting positions at the Imperial College of Science and Technology, London, the Systems and Control Group, Delft University, the MIT LIDS, Cambridge, MA, and at the Department of Aerospace and Mechanics, University of Minnesota, Minneapolis. His research interests include multivariable systems and robust control, system identification, fault detection with applications in power systems safety operations and also in the control of mechanical and vehicle structures. He is a fellow of the IFAC and the IEEE.

Akos Zarandy spent 27 years with the scientific research and development of various array processor architectures, implementations, real-time image processing problems, and applications. He obtained his PhD from the Hungarian Academy of Sciences in 1997. During his PhD studies, he spent more than 2 years at the University of California, Berkeley. He led several successful research and development projects, including onboard UAV vision system development and solved ultra-highspeed vision problems. He is a full professor at Pazmany Peter Catholic University from 2011. He is the author or co-author of 37 peer reviewed scientific papers published in international scientific journals. 